\title{
Velocity map imaging in time of flight mass spectrometry
}

\author{
M. Brouard, ${ }^{1, a)}$ E. K. Campbell, ${ }^{1}$ A. J. Johnsen, ${ }^{1}$ C. Vallance, ${ }^{1, b)}$ W. H. Yuen, ${ }^{1}$ and \\ A. Nomerotski ${ }^{2, c)}$ \\ ${ }^{1}$ The Department of Chemistry, The Physical and Theoretical Chemistry Laboratory, University of Oxford, \\ South Parks Road, Oxford OX1 3QZ, United Kingdom \\ ${ }^{2}$ Department of Physics, University of Oxford, Denys Wilkinson Building, Keble Road, Oxford OXI 3RH, \\ United Kingdom
}

(Received 8 September 2008; accepted 3 November 2008; published online 31 December 2008)

\begin{abstract}
A new variation on time of flight mass spectrometry is presented, which uses a fast framing charge coupled device camera to velocity map image multiple product masses in a single acquisition. The technique is demonstrated on two photofragmentation processes, those of $\mathrm{CS}_{2}$ and $\mathrm{CH}_{3} \mathrm{~S}_{2} \mathrm{CH}_{3}$ (dimethyldisulfide) at a photolysis wavelength of $193 \mathrm{~nm}$. In both cases, several mass fragments are imaged simultaneously, and speed distributions and anisotropy parameters are extracted that are comparable to those obtained by imaging each fragment separately in conventional velocity map imaging studies. () 2008 American Institute of Physics. [DOI: 10.1063/1.3036978]
\end{abstract}

\section{INTRODUCTION}

Since its development in the late $1980 \mathrm{~s},{ }^{1}$ ion imaging has become an extremely useful tool in reaction dynamics, ${ }^{2}$ with applications ranging from studies in dissociation dynamics ${ }^{3}$ through to inelastic scattering ${ }^{4,5}$ and chemical reactions. ${ }^{6,7}$ Over the intervening two decades there have been a series of innovations to improve the utility and resolution of the technology. These encompass both improvements in the instrumentation, for example, velocity mapping ${ }^{8}$ and slicing of the images, ${ }^{9-11}$ and in data acquisition programs, such as the introduction of centroiding ${ }^{12}$ and megapixelling ${ }^{13}$ algorithms.

These advances notwithstanding, one of the major limitations of conventional velocity map imaging is the relatively long time, on the order of milliseconds, required between capturing successive images, which allows only a single product mass to be studied per event. While not limiting for simple dissociation/collision processes, this makes the detailed study of more complicated systems, where there are likely to be a large number of different product channels, difficult, time consuming, and, in many cases, completely impractical.

Over the past few years, several methods have been developed to overcome this issue. One of the first was introduced by Tsai et al., ${ }^{14}$ and utilized a two dimensional ion detector, yielding a velocity distribution for each mass peak, but no angular information about the photofragments. More recently, Leskiw and co-workers ${ }^{15,16}$ employed an additional pulsed field to separate the flight paths of the different mass fragments in space, allowing them to be imaged onto separate portions of the detector. This allows full imaging of several mass fragments simultaneously, but is limited to frag-

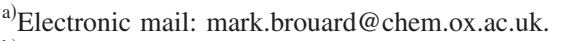

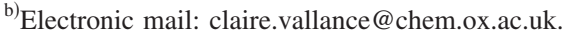

${ }^{c)}$ Electronic mail: A.Nomerotski1@physics.ox.ac.uk.
}

ments of similar mass, and would be difficult to apply to large numbers of different mass channels, as the required detection area becomes very large.

A further technology that allows the simultaneous imaging of several different mass fragments is the delay line detector, ${ }^{17}$ in which a wire mesh placed behind a microchannel plate is connected to a sequence of current sensitive detectors. Comparing the delays between different detectors registering the arrival of an ion allows an extremely accurate determination of the position of the particle's incidence on the detector. Such detectors have already been used successfully in coincidence experiments, ${ }^{18}$ and have the advantage of allowing detection of a theoretically unlimited number of mass channels. However, since the delay line measurement can only be made for one particle at a time, and requires several nanoseconds of dead time between each event, low signal count rates must be maintained for this approach to be successful. This problem is particularly acute where several peaks of different intensities need to be imaged simultaneously, as the most intense peak would be limited to a single ion incidence per event, making acquisition times for much less intense peaks extremely long.

The approach described in this paper, potentially overcoming many of the above difficulties, has been made possible by recent advances in silicon imaging technologies, which have led to the development of charge coupled device (CCD) sensors capable of acquiring and storing on chip a large number of images in quick succession. The aim of this investigation has been to use this new technology to combine time of flight mass spectrometry (TOF-MS) and ion imaging, with the resulting "imaging mass spectrometry" (Imaging MS) having advantages inherent to both.

The potential applications of such a method are diverse, and extend beyond the domain of reaction dynamics. For instance, combining the added velocity information of imaging with TOF-MS could be a useful tool for identifying large molecules such as proteins, for which mass spectra can be complex and often ambiguous. Modern mass spectrometers 
often rely on a "tandem" approach, in which mass-selected molecules are fragmented and the fragments characterized using TOF-MS. Fragmentation can be induced using any of a range of approaches, including collisionally induced dissociation, infrared multiphoton dissociation, electron capture dissociation, and UV photodissociation, with the fragmentation pattern being dependent on the method used. Imaging MS could potentially play an important role in these types of experiments, allowing the details of the dissociation process to be elucidated, and providing both mass and velocity information simultaneously. The technique may also find applications in molecular fingerprinting. In particular, the often highly characteristic velocity distributions arising from fragmentation may open the door to molecular fingerprinting of mixtures, a task that is generally beyond the scope of existing techniques.

To test the feasibility of this concept, a fast framing CCD camera, capable of recording on chip a series of 16 images with a time resolution of $10 \mathrm{~ns}$, has been combined with an existing, conventional velocity map imaging apparatus. ${ }^{19-21}$ Compared to conventional velocity map imaging, the main disadvantage of this new setup is the relatively low image resolution of the prototype fast framing camera (64 $\times 64$ pixels). Conventional CCD video cameras typically have a pixel resolution of around $500 \times 500 .{ }^{13}$ However, specialized CCD chips have already been developed that are capable of storing up to 100 images or more at resolutions comparable to standard $\mathrm{CCD},{ }^{22}$ and it is unlikely to be long before significant improvements on the prototype system described here will be realizable.

Two sets of experiments have thus far been performed with this new setup. The first, to illustrate the resolution and image quality of the new camera, involved imaging two products from the one photon $193 \mathrm{~nm}$ dissociation of $\mathrm{CS}_{2}$, specifically the $\operatorname{CS}\left({ }^{1} \Sigma^{+}\right)$and $\operatorname{S}\left({ }^{1} D_{2}\right)$ fragments. This yielded velocity distributions comparable in resolution to those obtained in with a conventional camera of much higher resolution $(576 \times 768$ pixels $)$ camera, imaging each peak separately.

The second experiment was carried out to investigate how well the method coped with imaging multiple fragments with very different quantum yields, via a study of the multiphoton fragmentation of dimethyldisulfide. Here we demonstrate that with imaging MS it is possible to obtain speed and anisotropy parameters that are in good agreement with a previous, higher resolution velocity map ion-imaging study. ${ }^{23}$

\section{EXPERIMENTAL}

The apparatus employed for imaging MS is essentially the same as that used for conventional velocity mapped imaging, and is described in detail elsewhere. ${ }^{24,25}$ A schematic of the experiment is shown in Fig. 1.

The target species is seeded in helium to a pressure of 2 bar, and expanded through a pulsed valve (General Valve) and a skimmer to form a molecular beam with a temperature of approximately $20 \mathrm{~K}$. Typical seed gas concentrations were $5 \%$ for the $\mathrm{CS}_{2}$ and $1.5 \%$ for the dimethyldisulfide experiments, respectively.

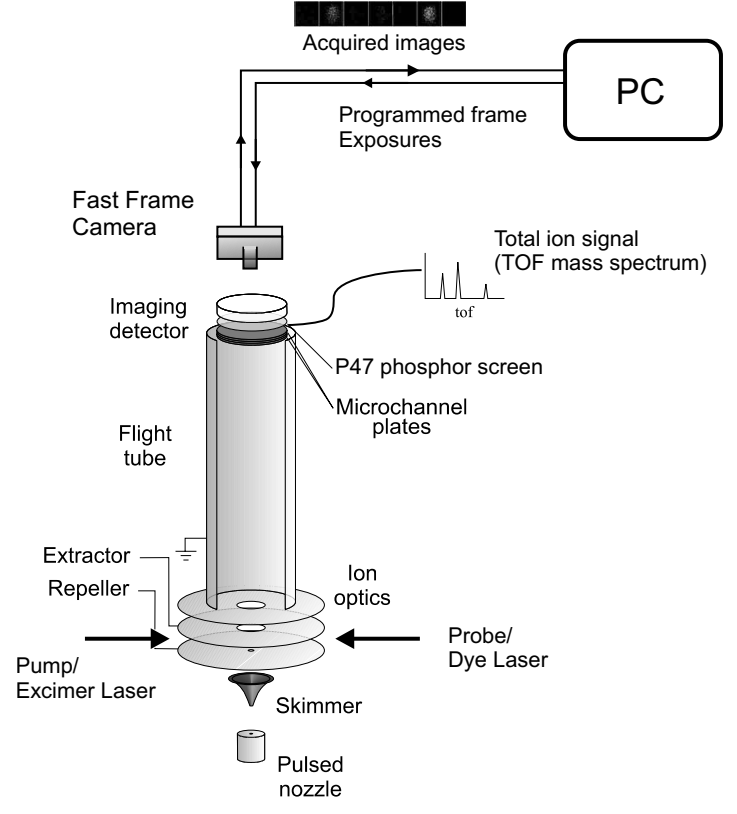

FIG. 1. Experimental apparatus. The main body of the instrument is essentially the same as that used in conventional velocity map imaging (Refs. 1 and 8).

The photofragmentation is performed at $193 \mathrm{~nm}$, using a focused ArF excimer laser with a pulse energy of approximately $0.5 \mathrm{~mJ}$. The photolysis products from $\mathrm{CS}_{2}$ are ionized using resonantly enhanced multiphoton ionization (REMPI). For dimethyldisulfide fragmentation and ionization occur via a number of multiphoton channels at $193 \mathrm{~nm}^{23}$

The resulting ions are velocity mapped onto a detector consisting of a pair of $75 \mathrm{~mm}$ diameter microchannel plates with a $32 \mu \mathrm{m}$ pore spacing, and a P47 phosphor screen (with a decay time of $\sim 120 \mathrm{~ns}$ ). The ion lenses are tuned such that the different masses arrive at the detector spaced in time by at least $250 \mathrm{~ns}$, in order to prevent overlap between consecutive images on the phosphor screen.

The camera used for this work is a programmable ultrafast frame transfer CCD camera (Dalsa Inc.), ${ }^{26}$ which can be clocked to the arrival times of the different masses. The camera is capable of recording and storing on chip up to 16 consecutive images with user defined exposure lengths after an initial trigger, with a time resolution of $5 \mathrm{~ns}$. The minimum and maximum exposure times for each image are $10 \mathrm{~ns}$ and $1.275 \mu \mathrm{s}$, respectively. After each acquisition cycle, the stored images are transferred from the chip to a PC at standard data rates for processing and storage.

In a multiple frame-transfer camera, ${ }^{22}$ each imaging pixel has a memory, implemented in a CCD register, to keep a considerable number of recorded "snapshots" inside the pixel during the data taking. This internal storage of images was made possible due to recent advances in the submicron silicon processing, and it allows for a remarkably fast operation of the camera limited only by the time of charge transfer between the neighboring CCD cells and not by the readout time of the full frame as before. When the camera is triggered (or "clocked"), the charge stored on the photosensitive part of the pixel is transferred into the first cell of the CCD register at the same time shifting the previously recorded 
cells by one. This charge transfer process in the registers occurs simultaneously across all of the pixels in the CCD chip. By synchronizing the clocking of the CCD chip to the arrival of consecutive mass peaks at the detector, images for each mass peak may be stored on the chip, up to a maximum number of images determined by the number of memory cells in the register within each pixel. The exposure time for each frame and the time required to transfer charge between registers may be as short as a few nanoseconds. Once the desired number of images has been stored, the complete set of data may be read out at a much slower rate (on the order of milliseconds) to a PC for processing.

The camera is programed with a set of 16 exposure times subsequent to the initial trigger. Once the trigger is received, the 16 consecutive images are acquired with the specified exposures. During the exposure the signal is continuously integrated by the sensor, so it is not possible to program dead time between the regions of the mass spectrum one wishes to image. In practice, exposure times of the masses of interest were kept constant, and intervening images were used as "junk images" or "padding" to achieve the required separation between the mass peaks of interest. For this reason, the maximum number of peaks that may be imaged is generally less than the number of frames that the chip can store.

\section{A. Image processing}

In order to minimize background signal a threshold was applied to the images, where any pixels with a brightness below this level were set to zero, and a limited form of event counting was implemented, in which any remaining nonzero pixel values were counted as single ions. The resulting images were then summed over 8000 laser shots.

In order to improve the image resolution, implementation of a centroiding algorithm ${ }^{12}$ was considered. However, a series of single shot acquisitions with the conventional and fast framing cameras, shown in Figs. 2 and 3, have revealed that the bright area on the detector from an incoming ion is considerably smaller than the area covered by each pixel of the fast framing camera, making the probability of an ion illuminating multiple pixels small. Centroiding would also carry the risk of miscounting two ions illuminating adjacent pixels, which is a more significant issue for this low resolution application.

After the images were acquired, they were analyzed using the Fourier moment analysis method, ${ }^{27}$ in order to extract speed distributions and speed dependent translational anisotropy parameters $[\beta(v)]$ from them. Although several other techniques are available for analyzing images, most notably the Abel inversion method, ${ }^{28}$ Fourier moment analysis, due to the inherent smoothing it performs for images with low resolution and signal levels, ${ }^{2}$ is particularly suited to this application. It also provides a very efficient method of storing image information, of potential use in providing a reference library for large-molecule fingerprinting studies. $\beta(v)$ is defined as

$$
\beta(v)=2\left\langle P_{2}(\cos \theta)\right\rangle,
$$

in which $\theta$ is angle between the velocity of the fragment and the transition dipole moment, $P_{2}(x)$ is a second order
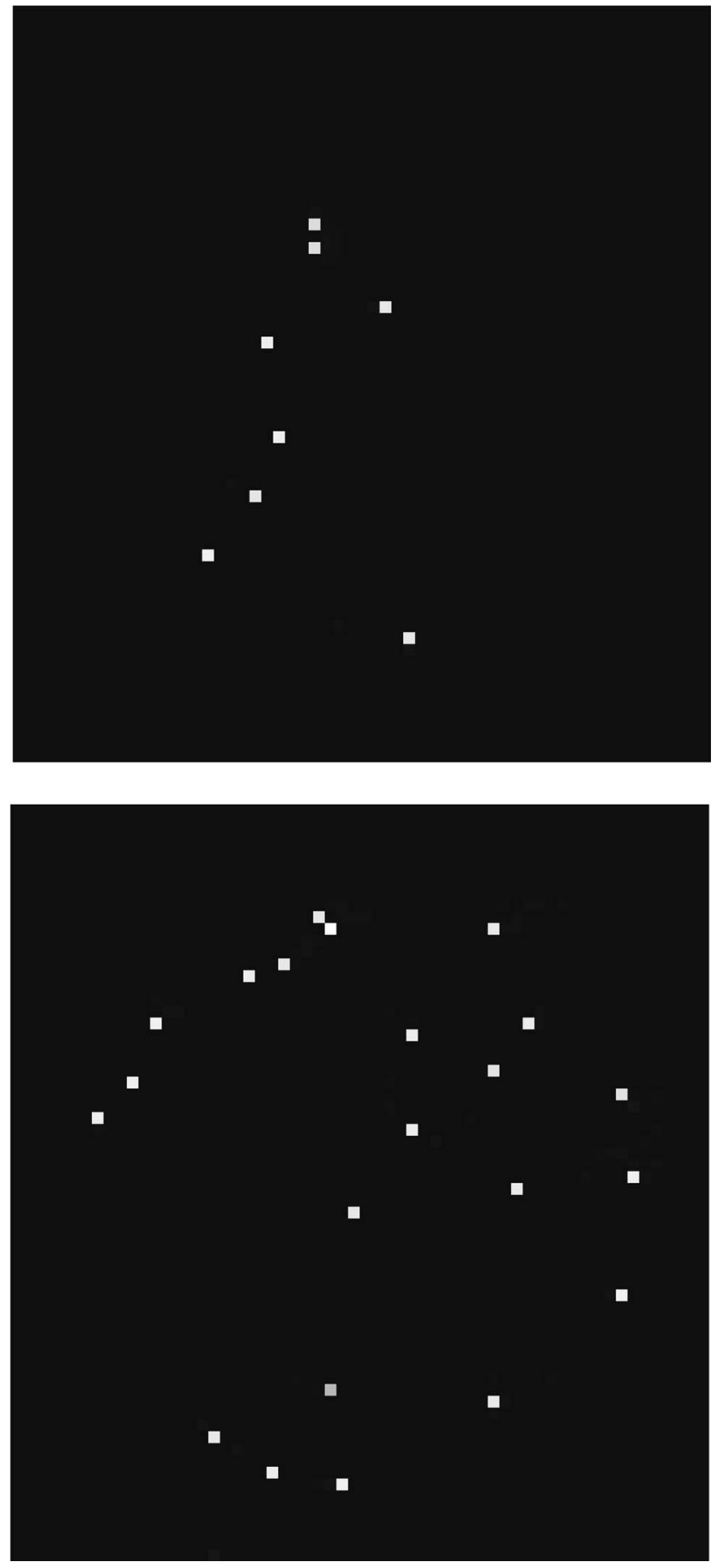

FIG. 2. Single shot images recorded with the fast framing CCD camera. Each ion only illuminates a single pixel, thereby limiting the benefits of using a centroiding algorithm (Ref. 12).

Legendre polynomial, and \langle\rangle denotes an ensemble average over $\theta$ at fragment speed, $v$. In principle, higher order anisotropy parameters, i.e., those pertaining to angular momentum polarization effects, are also obtainable, but would require acquisitions with several different pump/probe geometries. ${ }^{29}$

\section{RESULTS AND DISCUSSION}

\section{A. Carbon disulfide results}

Images of the CS and $S\left({ }^{1} D_{2}\right)$ fragments from the $193 \mathrm{~nm}$ photolysis of $\mathrm{CS}_{2}$ are shown in the left hand panels of Figs. 


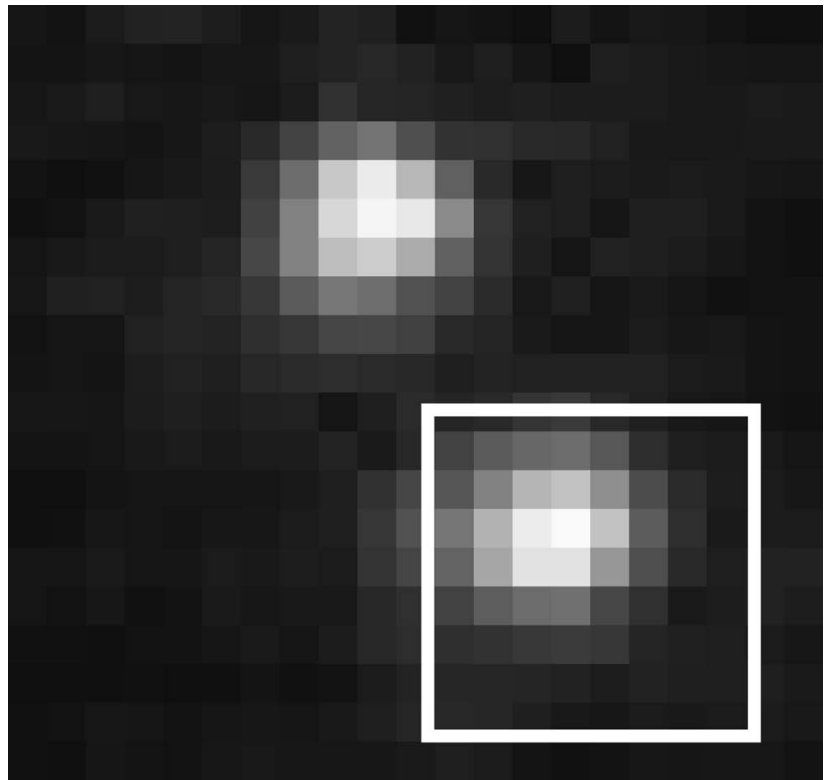

FIG. 3. An image of single ions obtained using a conventional (576 $\times 768$ pixels) CCD camera. The relative size of a single $100 \times 100 \mu \mathrm{m}^{2}$ fast framing pixel (white square) is provided for comparison.

4 and 5, respectively. To draw a comparison with conventional ion imaging, the experiments were repeated with a standard $576 \times 768$ pixel CCD camera, imaging each mass peak in the TOF spectrum in separate acquisitions. All other conditions were kept the same, as described in Sec. II.
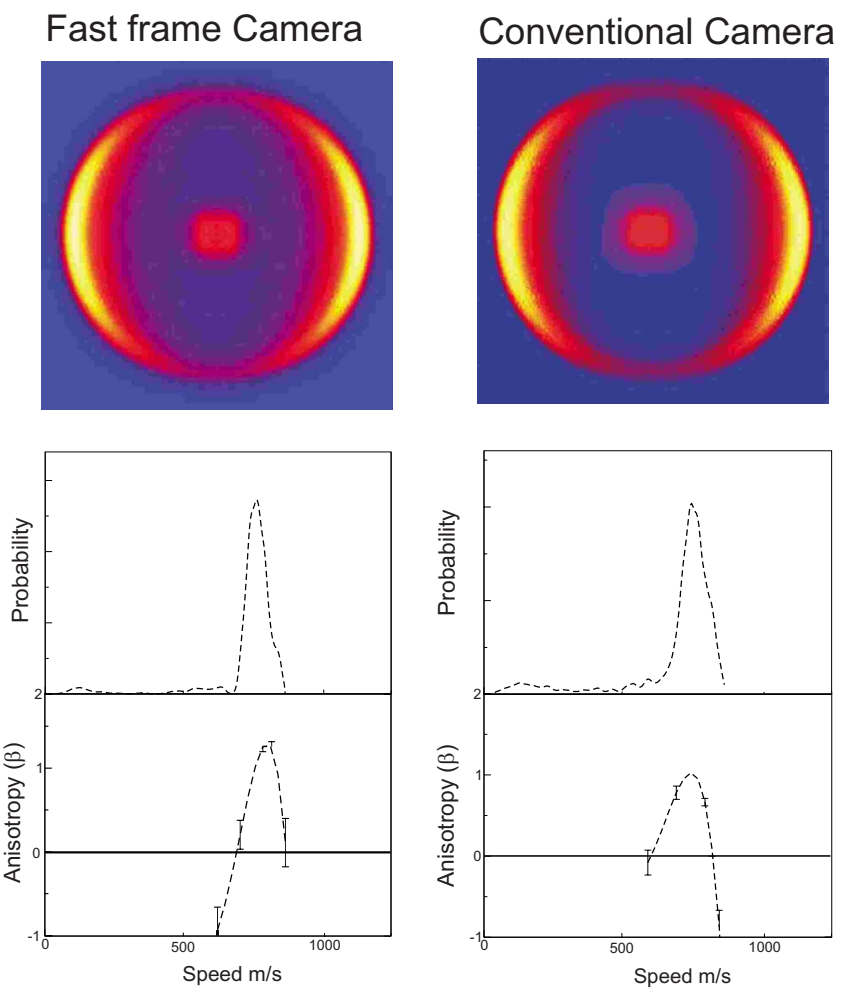

FIG. 4. (Color online) Comparison of CS fragment images from the $193 \mathrm{~nm}$ dissociation of $\mathrm{CS}_{2}$, obtained with fast framing and conventional CCD cameras (top panels). Speed distributions and $\beta(v)$ parameters derived from the images for both cameras are shown in the middle and bottom panels, respectively.
Fast frame Camera
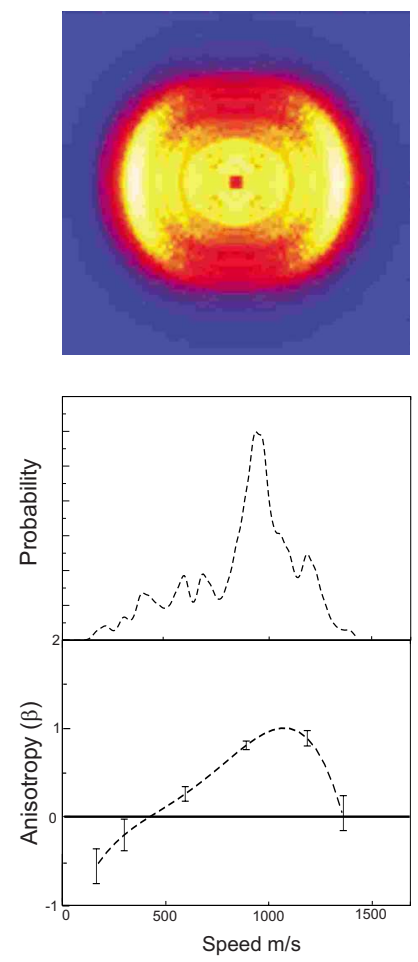

Conventional Camera
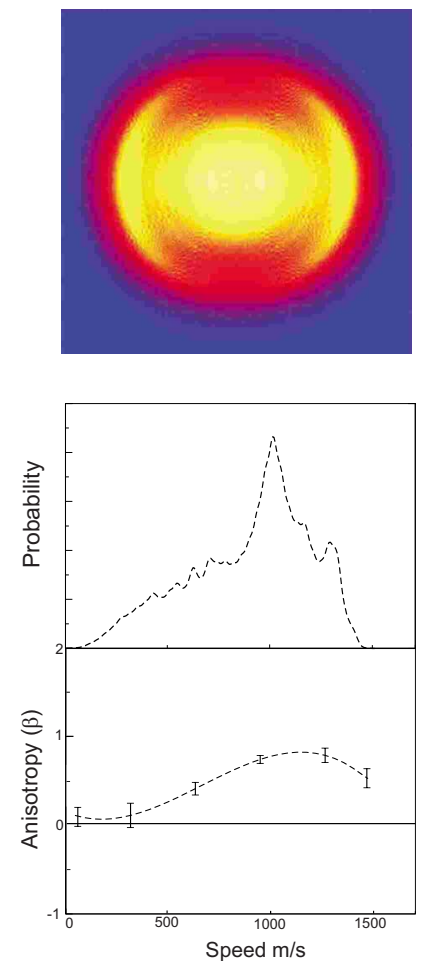

FIG. 5. (Color online) Comparison of $\mathrm{S}\left({ }^{1} D_{2}\right)$ fragment images from the 193 $\mathrm{nm}$ dissociation of $\mathrm{CS}_{2}$, obtained with fast framing and conventional cameras (top panels). Speed distributions and $\beta(v)$ parameters for both cameras are shown in the middle and bottom panels, respectively.

$\mathrm{CS}_{2}$ dissociation at $193 \mathrm{~nm}$ occurs via a one photon excitation from the ground $\tilde{X}\left({ }^{1} \Sigma_{g}^{+}\right)$to the bent ${ }^{1} \Sigma_{u}^{+}\left({ }^{1} B_{2}\right)$ state. ${ }^{30}$ The dissociation pathway is believed to be predissociative, with the initial excitation aligned parallel to the $\mathrm{C}-\mathrm{S}$ bond, and leading to the two exit channels:

$$
\begin{aligned}
& \mathrm{CS}_{2}+h \nu(193 \mathrm{~nm}) \rightarrow \mathrm{CS}\left(\Sigma^{+}, v_{\mathrm{CS}} \leq 13\right)+\mathrm{S}\left({ }^{3} P_{J}\right), \\
& \mathrm{CS}_{2}+h \nu(193 \mathrm{~nm}) \rightarrow \mathrm{CS}\left(\Sigma^{+}, v_{\mathrm{CS}} \leq 5\right)+\mathrm{S}\left({ }^{1} D_{2}\right) .
\end{aligned}
$$

Accessible vibrational states for the CS fragment range from $v_{\mathrm{CS}}=1-5$ and $v_{\mathrm{CS}}=1-13$ for the singlet and triplet channels, respectively, with a maximum total population at $v_{\mathrm{CS}}=3$.

In light of the above, images are anticipated that reveal a positive $\beta(v)$, together with significant rovibrational excitation in the diatomic fragment. Note that although there is expected to be a considerable yield of $S\left({ }^{3} P_{J}\right)$ atoms from the dissociation, only the $S\left({ }^{1} D_{2}\right)$ fragment was studied in the present work, as it is known to give a more structured velocity distribution. ${ }^{31}$

\section{CS fragment}

Due to the broad bandwidth of the excimer laser photolysis pulse, the $\mathrm{CS}^{+}$fragment image is believed to include contributions from several different rovibrational states, all arising from $1+1$ REMPI of neutral CS fragments at $193 \mathrm{~nm}^{32}$ 


$$
\begin{aligned}
\mathrm{CS}\left({ }^{1} \Sigma_{g}\right)+h \nu(193 \mathrm{~nm}) & \rightarrow \operatorname{CS}\left(A^{\prime}{ }^{1} \Sigma^{+}\right)+h \nu(193 \mathrm{~nm}) \\
& \rightarrow \mathrm{CS}^{+}+e^{-} .
\end{aligned}
$$

Nonetheless, its image, and associated velocity distribution, still exhibit relatively sharp structure.

Figure 4 compares the images, velocity distributions, and anisotropy parameters obtained using both the conventional and fast framing cameras. For this fairly simple distribution, it can be seen that the two sets of results are in very good agreement.

\section{2. $S\left({ }^{1} D_{2}\right)$ fragment}

The $S\left({ }^{1} D_{2}\right)$ fragment is ionized using a $2+1$ REMPI scheme via the ${ }^{1} F_{3} \leftarrow{ }^{1} D_{2}$ transition at $288 \mathrm{~nm}$ :

$\mathrm{S}\left({ }^{1} D_{2}\right)+2 h \nu(288 \mathrm{~nm}) \rightarrow \mathrm{S}\left({ }^{1} F_{3}\right)+h \nu(288 \mathrm{~nm}) \rightarrow \mathrm{S}^{+}+e^{-}$

using a pulse from a $\mathrm{Nd}$ :yttrium aluminum garnet pumped dye laser. ${ }^{25}$

Figure 5 shows a comparison between images, speed distributions and $\beta(v)$ parameters of the $\mathrm{S}^{+}$fragment for the conventional and fast framing cameras. The $\mathrm{S}^{+}$velocity distribution is determined by the rovibrational state distribution of the partnering CS fragment. We see from both sets of images that the speed distribution is relatively broad, and shows interesting structure both in the speed and angular coordinates. ${ }^{31}$ The results for the two cameras are again in good agreement with each other. However, it can be seen that, particularly at low velocities, where the lower pixel density of the fast framing camera becomes more significant, there are indeed some discrepancies between the speed distributions of the high and low resolution images, although the coarse shape of the distributions, and the trend in $\beta(v)$, remain the same.

\section{B. Dimethyldisulfide results}

Dimethyldisulfide fragmentation provides a different test of imaging MS to the photodissociation of $\mathrm{CS}_{2}$. In many ways, the system is closer to the anticipated applications for the imaging MS technique, as the photolysis process yields a variety of different mass channels. Although the images are considerably less structured than those resulting from the photolysis of $\mathrm{CS}_{2}$, the challenge here comes from the large variety of different image intensities arising from the branching ratios into the various product channels, all of which must be recorded simultaneously. There is a balance to be struck when choosing the laser power and microchannel plate gain, in order to obtain sufficient intensity for the weaker masses without saturating the detector on more intense peaks. In practice, when using event counting as described in Sec. II, this was straightforward to achieve, even with low count rates for the less intense peaks.

To demonstrate the advantage of this technique over conventional TOF mass spectrometry, a small amount of $\mathrm{CS}_{2}$ was added to the dimethyldisulfide, so that its $\mathrm{CS}^{+}$peak was still present in the spectrum. As $\mathrm{CS}^{+}$is also a potential fragmentation product of dimethyldisulfide, this could potentially

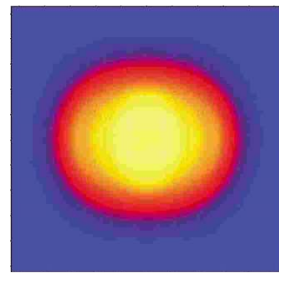

$\mathrm{S}^{+}$

Fragment

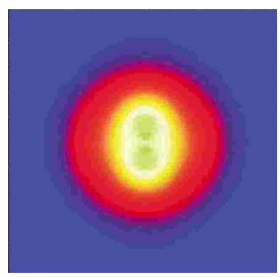

$\mathrm{S}_{2}^{+}$

Fragment

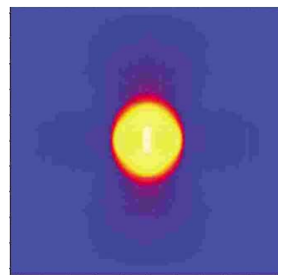

$\mathrm{MeS}_{2}^{+}$

Fragment

FIG. 6. (Color online) Images for the $\mathrm{S}^{+}, \mathrm{S}_{2}^{+}$, and $\mathrm{S}_{2} \mathrm{CH}_{3}^{+}$fragments from the photolysis of dimethyldisulfide at $193 \mathrm{~nm}$, collected with the fast framing CCD camera.

lead to confusion when identifying the spectrum. With the added dimension of imaging as well, however, the characteristic image arising from the "common" contaminant $\mathrm{CS}_{2}$ allows this peak to be assigned unambiguously.

In addition to the $\mathrm{CS}^{+}$contaminant peak arising from the photodissociation of $\mathrm{CS}_{2}$, images were also collected for the $\mathrm{S}^{+}, \mathrm{S}_{2}^{+}$, and $\mathrm{S}_{2} \mathrm{CH}_{3}^{+}$peaks, as shown in Figs. 6 and 7. Figure 7 provides a schematic of the multiple-mass imaging operation of the fast framing CCD camera. To assess the quality of our collected images, they are compared to a conventional imaging study performed previously on the same apparatus, under conditions similar to those described here. ${ }^{23}$

\section{Images, speed distributions, and anisotropy parameters for dimethyldisulfide}

Figures 8-10 compare the two sets speed distributions and $\beta(v)$ parameters for the $\mathrm{S}^{+}, \mathrm{S}_{2}^{+}$, and $\mathrm{S}_{2} \mathrm{CH}_{3}^{+}$fragments, respectively. As for the $\mathrm{CS}_{2}$ fragments, the conventional and

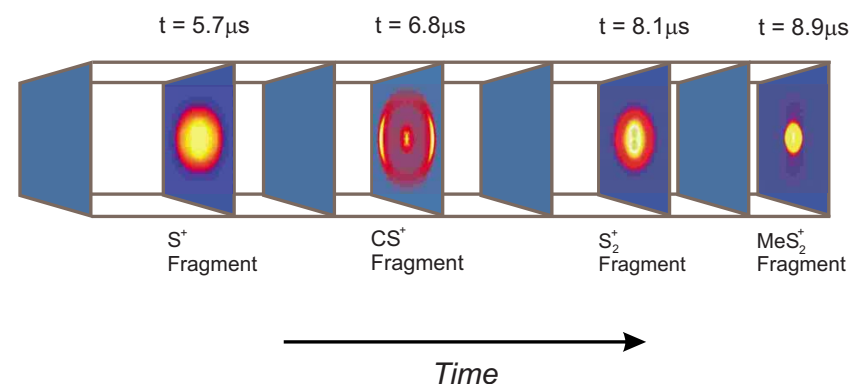

FIG. 7. (Color online) Schematic for the $\mathrm{S}^{+}, \mathrm{CS}^{+}, \mathrm{S}_{2}^{+}$, and $\mathrm{S}_{2} \mathrm{CH}_{3}^{+}$fragments from the photolysis of dimethyldisulfide and $\mathrm{CS}_{2}$ at $193 \mathrm{~nm}$, shown in sequence with their respective TOFs, and the blank spacing images between them. 


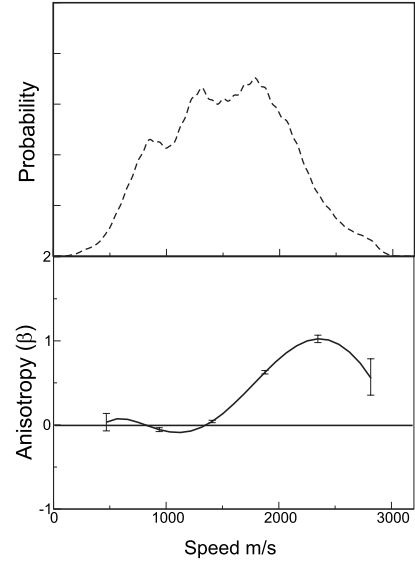

Fast Frame Camera

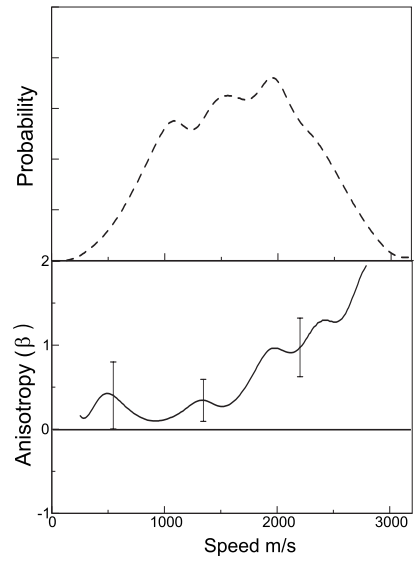

Conventional Camera

(b)
FIG. 8. Speed distribution and $\beta(v)$ parameter for the $\mathrm{S}^{+}$fragment from dimethyldisulfide photolysis at $193 \mathrm{~nm}$, obtained using the fast framing (a) and conventional (b) CCD cameras.

velocity map MS data are in good agreement. The experiments with the conventional camera were carried out several years ago, using a different experimental apparatus, and in this case some variation in the speed distributions is almost certainly attributable to the effect of a small difference in laser intensity. The ions are formed through a variety of different multiphoton processes, ${ }^{23}$ often with several pathways to the same product, and therefore variations in laser intensity can be expected to have a noticeable effect of the appearance of the images.

Figure 11 compares the conventional and imaging MS speed distributions and anisotropy parameters for the peak around the expected position for the $\mathrm{MeS}^{+}$fragment or the $\mathrm{CS}^{+}$fragment from the $\mathrm{CS}_{2}$ impurity. Though the masses of $\mathrm{CS}^{+}$and $\mathrm{MeS}^{+}$are slightly different, the two peaks could not be resolved in this set of acquisitions due to the much greater

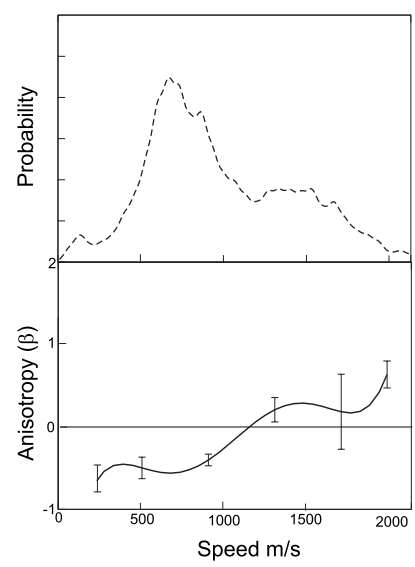

Fast Frame Camera

(a)

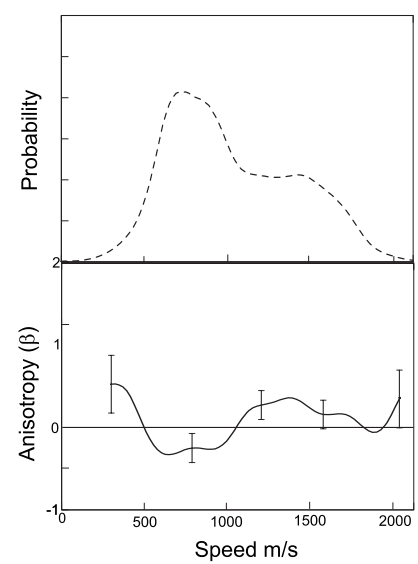

Conventional Camera

(b)
FIG. 9. Speed distribution and $\beta(v)$ parameter for the $\mathrm{S}_{2}^{+}$fragment from dimethyldisulfide photolysis at $193 \mathrm{~nm}$, obtained using the fast framing (a) and conventional (b) CCD cameras.

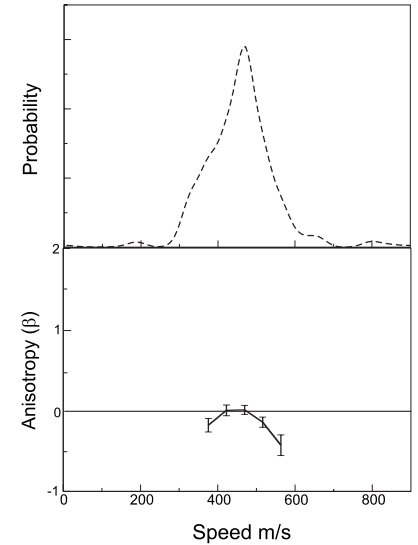

Fast Frame Camera

(a)

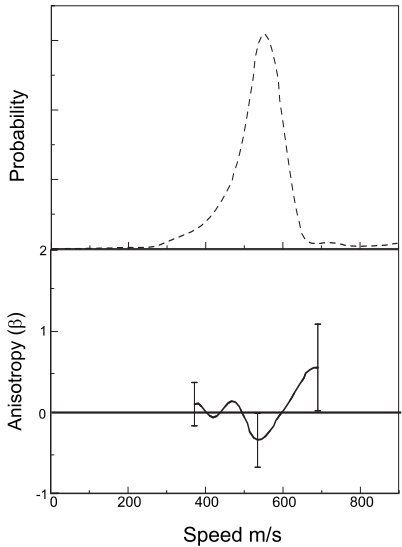

Conventional Camera

(b)
FIG. 10. Speed distribution and $\beta(v)$ parameter for the $\mathrm{MeS}_{2}^{+}$fragment from dimethyldisulfide photolysis at $193 \mathrm{~nm}$, obtained using the fast framing (a) and conventional (b) CCD cameras.

intensity of the $\mathrm{CS}^{+}$fragment, and the relatively long decay time of the phosphor screen employed ( $\sim 120 \mathrm{~ns})$. However, the data for the imaging MS are clearly in much better agreement with that collected for $\mathrm{CS}^{+}$than that of the $\mathrm{MeS}^{+}$from Ref. 23. This illustrates the potential fingerprinting that imaging can provide as a result of the additional information obtained for each ion signal.

The apparatus employed for these experiments was originally designed for highly velocity-resolved imaging, with mass resolution a secondary concern. However, methods to improve the mass resolution are available. For example, it is now possible to obtain relatively efficient phosphor screens with decay times as short as a few nanoseconds. It has also been demonstrated that it is possible to incorporate a reflectron into the velocity map ion-imaging experiment, ${ }^{15}$ thereby greatly enhancing the mass resolution.

\section{CONCLUSIONS AND FUTURE OUTLOOK}

We have presented a new method of performing mass spectrometry, in which velocity map imaging of each mass allows a wealth of additional information to be obtained for each TOF peak. The technique has been applied to the photofragmentation of $\mathrm{CS}_{2}$ and dimethyldisulfide, and, in both cases, images of sufficient quality to extract speed distributions and $\beta(v)$ parameters have been obtained for several mass fragments per acquisition.

The proof of principle experiments described here were carried out with a relatively low velocity (spatial) resolution fast framing CCD camera. However, this low velocity resolution, when compared to standard CCD, appears to be less crucial than anticipated, and at least for the systems studied so far $64 \times 64$ pixels seems sufficient to allow us to obtain a satisfactory picture of the photofragment dynamics. A more significant area to improve on in the future is the number of images collected per acquisition, as a true imaging MS technique must be capable of handling a large number of mass channels. Framing cameras already exist that are capable of taking 100 sequential frames, showing significant steps have 

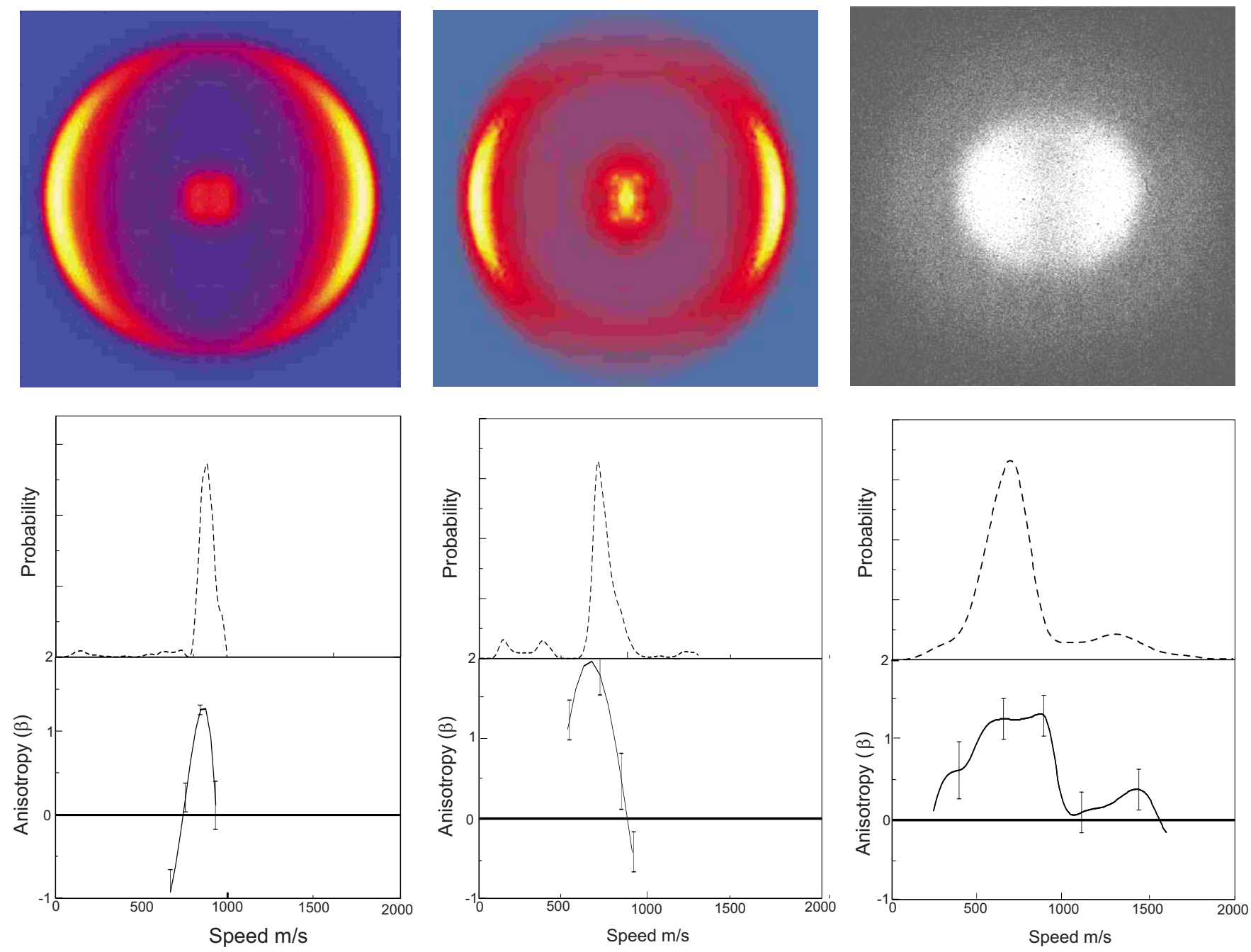

FIG. 11. (Color online) Comparison of ion images obtained on a mass peak around 45 amu using a conventional CCD camera, subsequent to photodissociation of $\mathrm{CS}_{2}$ (left) and dimethyldisulfide (right), with those obtained using the fast framing CCD camera following the photodissociation of dimethyldisulfide at 193 $\mathrm{nm}$ (top panels) in the presence of a small "contaminant" of $\mathrm{CS}_{2}$. Derived speed distributions and anisotropy parameters are shown in the middle and lower panels, respectively. In the conventional imaging experiment, the peak in the TOF spectrum at $45 \mathrm{amu}$ corresponds to the MeS ${ }^{+}\left(\mathrm{Ref}^{2} 23\right)$ fragment (right). The fast framing result (center), however, exhibits greater similarity to the $\mathrm{CS}^{+}$from $\mathrm{CS}_{2}$ dissociation (left).

been taken in this direction, and approaches relying on silicon pixel technology other than CCD may also be envisaged.

As it stands, the current apparatus will also allow the investigation of other systems in which multiple dissociation pathways are involved, which would be difficult to study using conventional velocity map imaging. Using VUV light to ionize the fragments should allow essentially "universal detection" 33 of different mass fragments. Together with a knowledge of their absorption cross sections this would, in addition to the added dynamical information from the imaging, allow branching ratios for different fragmentation processes to be determined.

It is hoped that in the future this technique can be extended to systems with a much larger number of mass fragments, and so provide a useful tool in reaction dynamics and analytical chemistry.

\section{ACKNOWLEDGMENTS}

The support from the EPSRC (to M.B. and C.V.) and a "proof of concept" grant from the ISIS Innovation Ltd. (to
M.B., C.V., and A.N.) were gratefully acknowledged. This work was subjected to a patent application from the ISIS Innovation Ltd. (Application No. 0724295.1).

${ }^{1}$ D. W. Chandler and P. L. Houston, J. Chem. Phys. 87, 1445 (1987).

${ }^{2}$ M. N. R. Ashfold, N. H. Nahler, A. J. Orr-Ewing, O. P. J. Vieuxmaire, R. L. Toomes, T. N. Kitsopoulos, I. A. Garcia, D. M. Chestakov, S.-M. Woo, and D. H. Parker, Phys. Chem. Chem. Phys. 8, 26 (2006).

${ }^{3}$ P. L. Houston, Acc. Chem. Res. 28, 453 (1995).

${ }^{4}$ A. G. Suits, L. S. Bontuyan, P. L. Houston, and B. J. Whitaker, J. Chem. Phys. 96, 8618 (1992).

${ }^{5}$ H. Kohguchi, T. Suzuki, and M. H. Alexander, Science 294, 832 (2001).

${ }^{6}$ T. N. Kitsopoulos, M. A. Buntine, D. P. Baldwin, R. N. Zare, and D. W. Chandler, Science 260, 1605 (1993).

${ }^{7}$ M. Ahmed, D. S. Peterka, and A. G. Suits, Chem. Phys. Lett. 301, 372 (1999).

${ }^{8}$ A. T. J. B. Eppink and D. H. Parker, Rev. Sci. Instrum. 68, 3477 (1997).

${ }^{9}$ C. Gebhardt, T. Rakitzis, P. Samartzis, V. Ladopoulos, and T. Kitsopoulos, Rev. Sci. Instrum. 72, 3848 (2001).

${ }^{10}$ D. Townsend, M. Minitti, and A. Suits, Rev. Sci. Instrum. 74, 2530 (2003).

${ }^{11}$ J. J. Lin, J. G. Zhou, W. C. Shiu, and K. P. Liu, Rev. Sci. Instrum. 74, 2495 (2003).

${ }^{12}$ B. Y. Chang, R. C. Hoetzlein, J. A. Mueller, J. D. Geiser, and P. L. Houston, Rev. Sci. Instrum. 69, 1665 (1998). 
${ }^{13}$ W. Li, S. D. Chambreau, S. A. Lahankar, and A. G. Suits, Rev. Sci. Instrum. 76, 063106 (2005).

${ }^{14}$ S. T. Tsai, C. K. Lin, Y. T. Lee, and C. K. Ni, Rev. Sci. Instrum. 72, 1963 (2001).

${ }^{15}$ B. D. Leskiw, M. H. Kim, G. E. Hall, and A. G. Suits, Rev. Sci. Instrum. 76, 104101 (2005).

${ }^{16}$ M. H. Kim, B. D. Leskiw, and A. G. Suits, J. Phys. Chem. A 109, 7839 (2005).

${ }^{17}$ Z. D. Pešić, D. Rolles, M. Perri, R. Bilodeau, G. Ackerman, B. Rude, A. Kilcoyne, J. Bozek, and N. Berrah, J. Electron Spectrosc. Relat. Phenom. 155, 155 (2007).

${ }^{18}$ M. Takahashi, J. Cave, and J. Eland, Rev. Sci. Instrum. 71, 1337 (2000).

${ }^{19}$ M. Brouard, A. Goman, S. J. Horrocks, A. J. Johnsen, F. Quadrini, and W.-H. Yuen, J. Chem. Phys. 127, 144304 (2007).

${ }^{20}$ M. Brouard, A. V. Green, F. Quadrini, and C. Vallance, J. Chem. Phys. 127, 084304 (2007).

${ }^{21}$ M. Brouard, F. Quadrini, and C. Vallance, J. Chem. Phys. 127, 084305 (2007).

${ }^{22}$ T. Etoh, D. Poggemann, G. Kreider, H. Mutoh, A. Theuwissen, A. Ruckelshausen, Y. Kondo, H. Maruno, K. Takubo, H. Soya, K. Takehara, T. Okinaka, and Y. Takano, IEEE Trans. Electron Devices 50, 144 (2003).

${ }^{23}$ B. Martinez-Haya, M. Bass, M. Brouard, C. Vallance, I. Torres, and J.
Barr, J. Chem. Phys. 120, 11042 (2004).

${ }^{24}$ M. Bass, M. Brouard, A. P. Clark, and C. Vallance, J. Chem. Phys. 117, 8723 (2002).

${ }^{25}$ M. Brouard, R. Cireasa, A. P. Clark, T. J. Preston, and C. Vallance, J. Chem. Phys. 124, 064309 (2006).

${ }^{26}$ See: http://www.dalsa.com.

${ }^{27}$ M. Brouard, R. Cireasa, A. P. Clark, G. C. Groenenboom, G. Hancock, S. J. Horrocks, F. Quadrini, G. A. D. Ritchie, and C. Vallance, J. Chem. Phys. 125, 133308 (2006).

${ }^{28}$ A. J. R. Heck and D. W. Chandler, Annu. Rev. Phys. Chem. 46, 335 (1995).

${ }^{29}$ A. S. Bracker, E. R. Wouters, A. G. Suits, and O. S. Vasyutinskii, J. Chem. Phys. 110, 6749 (1999).

${ }^{30}$ W. S. McGivern, O. Sokhabi, A. H. Rivi, A. G. Suits, and S. W. North, J. Chem. Phys. 112, 5301 (2000).

${ }^{31}$ T. N. Kitsopoulos, C. R. Gebhardt, and T. P. Rakitzis, J. Chem. Phys. 115, 9727 (2001).

${ }^{32}$ G. Dornhoefer and W. Hack, and W. Langel, J. Phys. Chem. 88, 3060 (1984).

${ }^{33}$ X. Yang, J. Lin, Y. T. Lee, D. A. Blank, A. G. Suits, and A. M. Wodtke, Rev. Sci. Instrum. 68, 3317 (1997). 